\title{
Barthélemy Boganda et l'Église catholique en Oubangui-Chari
}

Côme Kinata

\section{(2) OpenEdition}

1 Journals

Édition électronique

URL : http://journals.openedition.org/etudesafricaines/12292

DOI : 10.4000/etudesafricaines. 12292

ISSN : $1777-5353$

Éditeur

Éditions de l'EHESS

\section{Édition imprimée}

Date de publication : 20 septembre 2008

Pagination : $549-565$

ISBN : 978-2-7132-2184-2

ISSN : 0008-0055

\section{Référence électronique}

Côme Kinata, "Barthélemy Boganda et l'Église catholique en Oubangui-Chari », Cahiers d'études africaines [En ligne], 191 | 2008, mis en ligne le 20 septembre 2011, consulté le 19 avril 2019. URL http://journals.openedition.org/etudesafricaines/12292; DOI : 10.4000/etudesafricaines.12292

Ce document a été généré automatiquement le 19 avril 2019

(C) Cahiers d'Études africaines 


\title{
Barthélemy Boganda et l'Église catholique en Oubangui-Chari
}

\author{
Côme Kinata
}

1 Né le 9 avril 1910 à Bobangui dans la Lobaye, en pleine forêt équatoriale, Barthélemy Boganda fut très tôt orphelin et élevé par les missionnaires catholiques, avant de devenir le premier prêtre catholique oubanguien le 27 mars 1938. L'historien centrafricain Raphaël Nzabakomada-Yakoma (1986: 30), décrit ainsi les conditions historiques dans lesquelles Boganda vit le jour et passa son enfance :

«Ces territoires avaient subi, d'une manière générale, la même évolution depuis la fin du XIX siècle : d'abord des rapports antagonistes avec les Foulbé de l'Adamaoua, puis la soumission aux compagnies concessionnaires et à l'impôt de capitation à partir de 1893-1900, avec révoltes et répression, et enfin, en 1912, la domination allemande, en vertu du traité du 4 novembre 1911 par lequel la France, en échange de son protectorat sur le Maroc, cédait $25000 \mathrm{~km}^{2}$ de territoire de l'AEF à l'Empire Germanique. Devenus le Nouveau Cameroun, ces territoires participent, plus que toutes les autres colonies françaises, aux efforts de guerre et aux diverses opérations militaires, de 1914 à 1918. "

2 L'évangélisation de l'Oubangui fut conduite par Mgr Augouard qui, en février 1894, fonda la Mission Saint-Paul des Rapides à Bangui, puis en septembre 1894 la Mission SainteFamille des Banziri. Missionnaire de choc, il devança même les autorités coloniales dans la prise en main de la colonie, la parsemant très vite de chapelles de brousse qui précédèrent l'édification des bâtiments administratifs.

3 Un colonisateur et un missionnaire jouèrent un rôle important dans la vie de Boganda. Le colonisateur, le lieutenant Meyer, était administrateur de Mbaïki, qui, à l'époque, dépendait du Moyen-Congo. Le missionnaire, le père Herriau, de la congrégation du SaintEsprit, était supérieur de la Mission catholique de Bétou dont le champ d'apostolat s'étendait jusqu’à Mbaïki. Lors d'une tournée, Meyer recueillit le jeune Boganda dont le père venait d'être tué par des miliciens pendant une expédition punitive. Sa mère subit le même sort peu après. Dans ces conditions tragiques, Boganda, devenu orphelin, fut envoyé dans un orphelinat de fortune de Mbaïki improvisé par le père Herriau. 
4 Après la fermeture de la Mission de Bétou en décembre 1921, le père Herriau amena Boganda à la Mission Saint-Paul des Rapides à Bangui, où il fit sa scolarité. Nous retrouverons les détails de cette période dans la partie réservée à la formation. Mgr Augouard, l'une des figures emblématiques missionnaires de l'Afrique centrale, déclarait en 1890 à Poitiers lors d'une conférence: "Notre apostolat s'exerce essentiellement auprès des enfants, par eux nous conquérons l'Oubangui pour la France et pour Dieu.» Pendant près de dix huit ans, soit durant plus du tiers de sa vie, Barthélemy Boganda fut l'élève des missionnaires de la congrégation du Saint-Esprit. Ces mêmes missionnaires feront de lui un homme politique.

5 Grâce à Mgr Grandin, Barthélemy Boganda remplissait toutes les conditions et payait la caution pour se présenter aux élections législatives du $2^{\mathrm{e}}$ collège du 10 novembre 1946 à l'Assemblée de l'Union française. Le prélat, vicaire apostolique de Bangui expliquait ainsi son soutien : «On se lance en Oubangui : mais il y a tellement de gueulards anti-français que je n'ai pas hésité à lancer l'abbé comme adversaire aux Communistes, Socialistes, SFIO, etc., qui croient que nous sommes des moutons muselés »1. Boganda fut donc encouragé par son supérieur à faire de la politique, contrairement à son cadet l'abbé Fulbert Youlou du Moyen-Congo.

6 Devenu député, Boganda amorçait un autre versant de sa vie. Avec le temps, il devint un homme politique très influent dans la fédération des colonies qu'était l'Afrique équatoriale française (AEF). Il fut aussi le fondateur de la République centrafricaine. Il mourut dans un accident d'avion le 28 mars 1959, peu après avoir quitté Berberati. Lors de ses obsèques, le 3 avril 1959, le père Féraille - vicaire général à qui Boganda disait : "Vous êtes un des rares Spiritains pour lequel j'ai de l'estime $»^{2}-$, déclarait devant le parvis de la cathédrale de Bangui « qu'avant de devenir l'élu du peuple, Boganda avait été l'élu de Dieu » (Siango 2004 : 59). Dans les grands moments de sa vie politique, Boganda ne manquait jamais de rappeler la continuité de sa formation, de son sacerdoce et de son mandat politique. Il affirmait être investi d'une mission divine pour la libération et l'éducation de ses frères. La solidité de sa vocation fut, probablement, l'explication la plus plausible de sa force charismatique.

«Il fut le premier prêtre, le premier grand homme politique, le premier maire élu de Bangui, le Président du Grand Conseil de l'Aef (Afrique Équatoriale française) et enfin le fondateur de la République Centrafricaine le premier décembre 1958, quatre mois avant sa mort tragique » (ibid. : 9).

7 Nous étudierons d'abord la formation de Barthélemy Boganda et son ministère puis la rupture avec l'Église catholique.

La formation et le ministère sacerdotal de Barthélemy Boganda

8 La première partie de la vie de Boganda était entièrement placée sous la protection et la direction des autorités religieuses qui le recueillirent quand il avait huit ou neuf ans, le conduisirent à la prêtrise et à l'apostolat, puis le menèrent à la vie politique quand il eut trente-six ans.

9 C'est à la Mission Saint-Paul que Boganda reçut sa formation scolaire de base. Il devint chrétien le 24 décembre 1922 en recevant le baptême puis la confirmation le lendemain. Cette période, pendant laquelle il rencontra le père Calloc'h, eut une grande influence sur toute sa vie. Ce prêtre fut pour lui le modèle auquel il voulait ressembler. Boganda dit de lui plus tard:

«Premier de sa classe durant sa vie d'étudiant, philosophe remarquable, théologien averti, polémiste redoutable, il parlait le batéké et le lingala. Le ngbaka était sa 
langue habituelle. Il connaissait également le banda et le sango. Il était le dévouement et la bonté personnifiée sous un aspect austère de paysan breton, un être infatigable, un corps aussi dur que les roches de sa Bretagne. Cet homme, véritable anima sana in corpore sano, m'a vivement impressionné et marqué pour le reste de ma vie. Quatre années durant, tous les jours, les dimanches exceptés, il nous accompagnait, le fusil en bandoulière, sa pioche sur l'épaule, son coupe-coupe en mains, et, de $7 \mathrm{~h} 30$ à $10 \mathrm{~h}$, de $15 \mathrm{~h}$ à $17 \mathrm{~h} 45$, il travaillait la terre avec nous, à Yangoumara, comme nous et avec nous il vivait du produit de son travail. L'agriculture était son apostolat. Il nous a appris à compter sur nous-mêmes et à tirer notre subsistance de la terre, à attendre tout de notre travail. Une dizaine de Monseigneur Calloc'h aurait transformé l'Oubangui en 20 ans [...]. Calloc'h était pour nous la France et l'Église, l'esprit et le corps, en un mot l'homme complet » (Kalck 1995 : 42).

C'est à Mgr Calloc'h qu'il confia son désir de devenir prêtre. «J'étais cependant inquiet car je n'avais jamais entendu dire qu'il existait des prêtres de ma couleur », racontait-t-il plus tard'. Mgr Jean-René Calloc'h remarquait l'intelligence de l'enfant Boganda et surtout son extraordinaire volonté. Cet enfant se remettait lentement des maux dont il était atteint. Le jeune Ngbaka avait trouvé dans Mgr Calloc'h un véritable parent adoptif. Disposant de peu de moyens pour le fonctionnement de leurs Missions, les missionnaires étaient contraints de travailler eux-mêmes et de faire travailler leurs élèves. Les travaux manuels auxquels le jeune orphelin dû participer jouèrent un rôle éminent dans sa formation. Une trentaine d'heures de travail dans les plantations ne laissait guère de place à l'enseignement proprement dit, au catéchisme et à l'étude. Il fallait que le petit orphelin de la forêt soit particulièrement surdoué pour qu'en moins de trois ans il accède au baptême, à la maîtrise du français et accomplisse le cycle complet des études primaires. Baptisé à Noël 1922, Boganda écrivit plus tard ce qu'il en pensait :

«Le 24 décembre 1922, je devenais chrétien. J'avais douze ans. Être chrétien signifiait pour moi s'émanciper des coutumes ancestrales, devenir frère de l'humanité. J'ai été tellement déçu depuis. Le 25 décembre, après la première communion et la confirmation, je me présentai chez Mgr Calloc'h pour lui exprimer ma volonté de faire tout ce qui dépendait de moi, de me soumettre à toute sorte de discipline, pour pouvoir, un jour, sortir mon pays et mes frères de la situation où ils se trouvaient. Une seule porte pouvait m'être ouverte rendant possible cet idéal : le sacerdoce. Encore, je n'en étais pas sûr, n'ayant jamais entendu dire qu'il existait des prêtres de ma couleur. Je me hasardai : "Monseigneur, je veux travailler pour mon pays et mes frères." Il connaissait déjà mes idées et mes dispositions. La réponse fut sèche : “C'est dur et c'est très long." J'ai timidement répliqué : "Je serai dur pour moi-même et je mettrai les bouchées doubles." Et je le fis » (ibid. : 43).

En juillet 1924, Boganda avait achevé sa scolarité à Saint-Paul. Il n'existait dans la colonie aucune école secondaire. Le père Fayet s'employait à réunir des fonds auprès des familles de la métropole. Grâce à ces subsides, le jeune écolier pouvait, en novembre 1924, embarquer sur le Pie x, vapeur des missionnaires, pour se rendre à Brazzaville. De là, il traversait le Pool pour gagner Kinshasa. Il entrait au petit séminaire de Kisantu, dirigé par des jésuites belges. D'octobre 1928 à juillet 1930, il fit sa troisième et sa seconde au petit séminaire de Brazzaville en compagnie de Auguste Roch Nkounkou et de Eugène Nkakou, les deux premiers prêtres indigènes du vicariat apostolique de Brazzaville. Ce séminaire était ouvert par Mgr Augouard, celui-là même qui avait été dépêché de Landana (sur la côte atlantique) pour veiller sur le pavillon français à Mfoa (future Brazzaville). 
Contrairement à Mgr Carrie ${ }^{4}$ du vicariat de Loango, qui ouvrit presque en même temps des écoles primaires et des séminaires, Mgr Augouard crut bon de multiplier d'abord des baptisés, des confirmés, des écoles primaires et autres bonnes œuvres avant d'ouvrir un petit séminaire. «Pour Mgr Augouard, l'Afrique est un continent maudit, entièrement soumis à Satan; il est sauvage, barbare. Les indigènes sont les enfants du démon, victimes de toutes les tares, non seulement celles qui choquent particulièrement les occidentaux, comme l'anthropophagie et la polygamie, mais aussi le polythéisme idolâtre, le tribalisme belliqueux, la tyrannie de l'esclavagisme, l'alcoolisme omniprésent, la paresse au travail, etc. ( (de Banville 2000 : 37). Il fallait donc régénérer l'Afrique en mettant en œuvre des entreprises pour créer un nouveau type d'hommes. Étant donné que les adultes ne pouvaient être convertis au christianisme, il fallait s'occuper des enfants que l'on pouvait isoler et former «en vase clos" selon les principes moraux de l'Évangile. Et comme, au début, les seuls enfants disponibles étaient des enfants esclaves, capturés durant les guerres tribales entre voisins, une partie de la méthode missionnaire de cette époque se traduisit par le rachat d'enfants esclaves (ibid.). fondation de la Mission de Brazzaville (1888). Il occupait des bâtiments situés dans l'enceinte de la cathédrale du Sacré-Cœur. Les candidats au sacerdoce furent nombreux, mais très peu purent continuer. Et pour cause! Mgr Augouard s'en explique dans son dernier rapport à la Sacrée Congrégation de la Propagande en 1920 :

«Plus de 7 élèves sont déjà passés par ce séminaire mais, hélas! Les réussites n'ont pas correspondu à nos efforts. La plupart de ces enfants sont morts d'anémie ou par suite de la terrible maladie du sommeil. À ces petites natures sauvages, la liberté semble nécessaire pour vivre. D'autres enfants se découragent par suite de la longueur des études et sont effrayés des quinze et vingt ans nécessaires pour parvenir à la prêtrise. L'instruction spéciale qu'ils ont reçue cependant n'est pas perdue, car beaucoup de ceux-là deviennent d'excellents catéchistes et sont pour nous de précieux auxiliaires.

D'autres enfin sont poussés par des parents païens et âpres au gain et nous quittent pour entrer comme écrivains dans l'Administration ou deviennent téléphonistes, chefs de gare. Généralement, ils font honneur à la mission qui les a élevés. Cependant, nous poursuivons cette œuvre importante du Séminaire où nous avons en ce moment (1920) 16 élèves, et nous espérons que Dieu voudra bien faire germer la semence que nous jetons péniblement dans les âmes. Nous espérons aussi que, selon les instructions du Saint-Père, l'œuvre de la Sainte-Enfance à Paris voudra bien nous aider à entretenir ce Séminaire d'où dépend l'avenir de la Mission. Toutefois, il ne faut pas dissimuler qu'il est difficile de faire germer des vocations au milieu du bourbier du paganisme, et du reste les Noirs ont beaucoup plus de confiance dans le prêtre européen que dans leurs propres congénères, contrairement à ce que l'on croit généralement $\|^{5}$.

Cette œuvre de Mgr Augouard végéta pendant longtemps :

«Elle était restée comme noyée dans un complexe d'œuvres annexes qui entravaient, retardaient, et annihilaient jusqu'à un certain point, sa marche normale. D'autre part, la vie intérieure souffrait de la proximité de la grande agglomération brazzavilloise dont tous les échos se prolongeaient jusque dans l'enceinte sacrée $»^{6}$.

Le 29 avril 1936, Mgr Biéchy, évêque de Brazzaville, décida de transférer rapidement ce séminaire à Kibouende. Cette localité, située à $53 \mathrm{~km}$ de Brazzaville sur la ligne du chemin de fer Congo-Océan, fut choisie en raison de la facilité du ravitaillement et du contrôle épiscopal. Le 26 septembre 1936, onze petits séminaires s'installèrent à Kibouende. 
Les 12 et 13 février 1937, Mgr Biéchy bénit l'oratoire et le séminaire tout entier. L'œuvre fut placée sous le patronage du grand apôtre saint Paul et le 25 janvier était désormais le jour de fête du petit séminaire ${ }^{7}$. Les effectifs du séminaire, au moment du transfert à Kibouende, étaient de deux théologiens, les abbés Auguste Nkounkou et Eugène Nkakou se rendant à Libreville terminer leur dernière année de théologie, et l'abbé Jean-Baptiste Olomadzanga devant être tonsuré à Yaoundé le 30 mars 1937, tandis que Fulbert Youlou entreprenait la deuxième année de philosophie à Yaoundé. Théophile Mbemba fut rapatrié pour des raisons de santé. Pour lui, on cherchait encore un pays au climat plus favorable pour qu'il continuât ses études. Ainsi, sur place, n'étaient restés que sept élèves dont trois en seconde, trois en quatrième et un seul en sixième. À Kibouende, grâce à « l'éloignement de la ville, des efforts sérieux et méritoires ont été faits dans la voie du silence et du recueillement $»^{8}$. Des études serrées donnèrent des résultats tangibles et des progrès inespérés. Mais en 1939, on décida de transférer le petit séminaire de Kibouende à Mbamou. Pourquoi ? «La seule raison connue semble être la difficulté d'entente entre le directeur de la station et le directeur du petit séminaire $»^{9}$. Mbamou, situé à $60 \mathrm{~km}$ au sud-ouest de Brazzaville, est enclavé ; une route sablonneuse le relie à la gare du chemin de fer Congo-Océan sur $20 \mathrm{~km}$. Ces difficultés d'accès furent un obstacle majeur pour ses rapports avec l'extérieur. Malgré tout,

«Mbamou est un endroit heureux. Émergeant au milieu de la verdure de nombreux palmiers et arbres fruitiers, au sommet d'un gentil plateau, on y trouve un bâtiment qui sert de résidence aux professeurs. Derrière, c'est le séminaire proprement dit. Il est simple, mais coquet, inspirant l'idée d'ordre et de propreté $»^{10}$.

17 Autant que possible, on plaçait le séminaire assez loin des autres centres européens pour que les élèves n'aient pas constamment sous les yeux l'exemple de leurs camarades pourvus d'emplois bien rénumérés. Avant l'installation du séminaire, Mbamou avait déjà une histoire : créé en 1896 par la Mission Marchand, il était à la fois poste militaire et poste administratif jusqu'en 1911, date à laquelle on transféra ces fonctions à Kinkala. Après ce transfert, Mgr Augouard envoya en septembre 1911 les pères Rémy et Patron pour trouver l'emplacement d'une Mission chez les Bakongo. Cette Mission devait être placée à proximité de la petite ligne de chemin de fer Brazzaville-Mindouli, et non loin de la future ligne Loango-Brazzaville. Le choix se fixa sur une colline qui dominait l'ancien poste français de Mbamou. Sur cette colline s'étendait un plateau qui avait dû être habité dans le passé, car les palmiers y abondaient. Le père Patron, aidé par le père Bonnefont, transportait à la nouvelle Mission le matériel de Kialou (à $90 \mathrm{~km}$ au nord de Kindamba). Les anciens bâtiments du poste français, situés à 7800 mètres du nouvel emplacement, dispensèrent de loger sous la tente ${ }^{11}$.

18 La formation du clergé à Mbamou fut une véritable révolution après la Seconde Guerre mondiale à tous les points de vue, du recrutement au programme, en passant par les techniques de formation. La formation au petit séminaire de Brazzaville fut ainsi décrite par le père Jean Morizur (1948: 156), directeur du petit séminaire de Mbamou, pour justifier les progrès réalisés par l'établissement qu'il dirigeait :

« Nous ne nous trouverons plus devant un embryon de maison de formation, où l'on prépare cinq ou six futurs prêtres entre deux tournées de brousse, les munissant d'un si faible et si hétéroclite bagage de connaissances que nous sommes les premiers, plus tard, à nous plaindre de la limite de leurs horizons. "

Le séminaire de Mbamou était intervicarial, recevait des élèves venus de tous les vicariats de l'AEF. Ainsi, Mgr Joachim N’Dayen, archevêque émérite de Bangui, fut élève à Mbamou. L'enseignement des sciences modernes était fonction de la compétence du professeur, ce 
qui n'était pas souvent le cas car les missionnaires venaient prioritairement pour l'évangélisation pure, et moins pour un enseignement profane approfondi en dehors de la littérature. Ainsi, le séminariste était par vocation un littéraire et recevait en outre des enseignements en rapport avec le culte : musique, chant, liturgie de diverses cérémonies. Le sport lui était particulièrement conseillé. Les deux premiers élèves de Brazzaville furent envoyés au petit séminaire de Lemfu, près de Kisantu, au Congo belge, puis au Gabon, en 1931. Il s'agit des abbés Auguste Roch Nkounkou et Eugène Nkakou. Pendant ce temps, Barthélemy Boganda enseignait le catéchisme à la Mission Saint-Paul à Bangui. Mgr Calloc'h démissionna de son poste en 1926. Il fut remplacé par Mgr Grandin pour qui Boganda avait une grande estime et un profond respect. En 1931, Mgr Grandin envoya Boganda à Mvolye au Cameroun. Fulbert Youlou, futur président de la République du Congo, y était son condisciple. Ordonné prêtre le 27 mars 1938, Boganda dit sa première messe le lendemain à Saint-Paul des Rapides à Bangui, à l'endroit même où il fut baptisé.

Boganda exerça son ministère sacerdotal d'abord à Saint-Paul à Bangui par des messes, des prédications, des confessions, puis comme professeur et directeur au petit séminaire Saint-Marcel de Bangui, assurant ainsi la marche vers le sacerdoce des pionniers comme François Ngaïbi, le premier prêtre tchadien. Il évangélisa également à Zongo, localité située en face de Bangui sur la rive belge. Il commença par enseigner le catéchisme, entendre les confessions et dire la messe sous un hangar. Ne supportant pas cette situation inconfortable, il bâtit une chapelle sans en référer à Mgr Grandin, son évêque. Il fit seulement appel au cœur et à la raison de ses frères du Congo belge qui mirent la main à la pâte. Boganda ne tint pas compte de la volonté du souverain pontife qui, quelques années plus tôt, avait interdit au père Augouard, missionnaire français, d'évangéliser en territoire belge. En 1942, Boganda fut affecté à Bambari où il eut une responsabilité de plus : une école supérieure venait d'être créée à Bambari. Il eut la charge d'y préparer les élèves au baptême et à la confirmation. Ces catéchumènes n'étaient pas tous aspirants au baptême, certains étant déjà chrétiens. Mais selon l'opinion de tous, les cours de catéchisme de Boganda étaient des événements à ne pas rater. En dehors du grand centre de Bambari, Boganda affectionnait particulièrement les postes secondaires comme Grimari.

Ce fut pendant son ministère sacerdotal que Boganda vécut la ségrégation raciale des Spiritains. Il accumula des rancœurs qui explosèrent après la mort accidentelle de Mgr Grandin. Mgr Cucherousset, qui remplaça ce dernier, ne fut pas tendre avec Boganda. Une véritable cabale fut montée contre le seul prêtre indigène de l'Oubangui. La rupture ne tarda pas.

La rupture avec l'Église catholique

22 Le christianisme, propagé par les missionnaires à l'époque coloniale, qui contribua largement à asseoir l'hégémonie française et la puissance coloniale, possédait sa propre contradiction interne: la parole salvatrice se trouvait minée de l'intérieur par l'ethnocentrisme et la domination du Blanc. L'attitude du clergé missionnaire à l'égard de la culture africaine fut faite d'incompréhension, de mépris et de rejet de la culture autochtone. Tout cela malgré les instructions du pape :

«Les missionnaires veilleront à éviter d'introduire parmi les populations en cours d'évangélisation les lois et usages particuliers à leur patrie personnelle [...]. Ils auront grand soin au contraire qu'en tout et partout ce soit la discipline ecclésiastique telle qu'elle est en vigueur dans l'Église universelle qui soit introduite et fidèlement observée. Les missionnaires auront également souci de bannir toute idée de préparer la voie parmi les populations qui leur sont confiées à une 
pénétration politique de leur nation, afin de ne pas passer pour rechercher le bien de leur patrie terrestre, non celui de Jésus-Christ et du royaume céleste ${ }^{12}$. en 1659 : « Ne mettez aucun zèle, n'avancez aucun argument pour convaincre ces peuples de changer leurs rites, leurs coutumes et leurs mœurs, à moins qu'ils ne soient évidemment contraires à la religion et à la morale. " Discrimination qui suppose la connaissance. C'est pourquoi les bons missionnaires ont toujours été de bons ethnologues ${ }^{13}$. Non seulement ils étaient conscients des aspirations des peuples, aspirations surgies du mode de vie local ou du besoin plus universel de salut, mais ils reconnaissaient dans les modes de vies variés les canaux par lesquels ces aspirations s'exprimaient et par où passaient les réponses à ces aspirations (Nida 1954: 120). Mais si une telle attitude rencontrait théoriquement une approbation quasi universelle en ce qui touchait le domaine profane d'une culture, la même unanimité était loin de se réaliser quant au domaine religieux. Nous comprendrons mieux cette situation en regardant de plus près la situation en Oubangui-Chari à travers celle vécue par Barthélemy Boganda.

«L'Abbé Boganda exercera son sacerdoce pendant huit années, dont trois à la mission Saint-Paul des Rapides à Bangui et cinq dans la Ouaka, en plein pays Banda. Il constituait, à lui seul, le clergé indigène d'Oubangui-Chari. Ni religieux, ni missionnaire, il avait pleine conscience de sa place dans l'avènement d'une Église oubanguienne. Dans les comptes rendus qu'il adressait à son évêque, il ne cachait rien de ses divergences avec les missionnaires » (Kalck $1995: 53$ ).

Le pays banda était réputé pour sa résistance aux chasseurs d'esclaves venus du Darfour, du Ouaddaï, du Bahr el Ghazal. Les nombreuses communautés banda s'étaient répandues dans les savanes oubanguiennes, poussant même jusqu'à la grande forêt. La cohésion de ce peuple tenait précisément à un attachement farouche à des coutumes perpétrées grâce à des sociétés d'initiation fort actives.

Comme partout en Oubangui, les sociétés concessionnaires avaient causé dans la région de graves ravages alors que celle-ci n'était pas encore remise de ses combats contre les chasseurs d'esclaves. La résistance aux nouveaux arrivants, et notamment à la collecte de l'impôt, avait nécessité de sévères répressions militaires. Pour recruter ses porteurs, Émile Cointet utilisait « cinquante mètres de forte chaîne et une solide chicotte [...]. Après avoir obéi au début et surtout par curiosité, ils commencent à en avoir assez [...]. Ce sont des populations très primitives et très craintives, mais si on ne sait pas les prendre, ils feront ce qu'ils ont déjà fait: le vide autour de nous $»^{14}$. Comme pour le portage, la population se dispersa devant les percepteurs de l'impôt.

"Vous arrivez dans un village et, après deux ou trois heures de pourparlers, pour se débarrasser de vous, on vous donne généreusement une poule ou quelques kilos de poissons secs en promettant, car on promet toujours que la prochaine fois on paiera le tout. Et, quand vous repassez deux mois après, dès que vous êtes signalés, tout le village s'enfuit dans la brousse: vous ne trouvez qu'un homme ou deux qui vous disent qu'on est au caoutchouc et qu'on paiera au retour $»^{15}$.

Voilà les conditions qui avaient traumatisé les populations avec qui devait travailler Barthélemy Boganda. Cet abbé indigène, décidé à évangéliser ses congénères par une méthode nouvelle, "évangélisation par l'école, l'école pour l'évangile». Ce principe souleva la raillerie des Blancs, dont son curé, le père Kandel. Pour les Blancs, la mission envisagée par Boganda exigeait un optimisme peu ordinaire. Ce à quoi Boganda répliquait qu'il ne s'agissait pas d'optimisme et qu'il avait foi dans sa mission divine. À propos de ces difficultés, le père Kandel a cru devoir lui dire : «Fermez les yeux si vous ne voulez pas 
vous créer de graves difficultés avec l'administration $»^{16}$. L'administrateur Dieu imposait le respect des coutumes, abjectes pour l'abbé Boganda: polygamie, fétichisme, divorce étaient des coutumes à combattre. De tout temps et partout, ce principe du respect des coutumes a toujours été la pomme de discorde entre administrateurs et missionnaires (Kinata 2004 : 593).

Les administrateurs parlaient en termes d'évolution, les missionnaires en termes de conversion. Dans le journal de la Mission de Grimari, à la date du 24 février 1943, on pouvait trouver ce commentaire de la main de l'abbé Boganda :

«Mon catéchiste de Grimari, Thomas Kola, m'apprend que le commandant ne veut pas que "les gens du mariage" reconstruisent leur village. On se rend aussitôt chez M. Dieu qui nous reçoit d'ailleurs assez fraîchement. "Je suis en train d'écrire, dit-il, à $\mathrm{M}$. l'Abbé qui s'est permis de régler ici les palabres à sa façon, sans tenir compte des coutumes indigènes." Cela parce qu'à mon dernier voyage, j'ai fait rentrer au foyer paternel des jeunes filles qui vivaient avec des polygames et dont la plupart avaient été livrées de force par leurs parents. On dit: "Nous venons donner la civilisation aux indigènes." Civilisation chrétienne et coutumes indigènes, voilà des contradictions, à mon avis, moi qui suis indigène. Coutumes indigènes signifie religion et cette religion c'est le fétichisme; coutume indigène, c'est le mariage forcé qui est une transformation très récente de l'ancien esclavage. "

L'abbé tentait avec la faiblesse de ses moyens de développer l'œuvre d'éducation en luttant contre les coutumes précitées. Il se heurtait tant à la résistance des familles restées attachées à l'initiation traditionnelle qu'à celle de l'administration. De Grimari, l'abbé Boganda rappelait à son évêque que son action religieuse était inséparable de son action sociale.

«À mon ordination sacerdotale, je me suis mis entièrement entre vos mains pour travailler à l'évangélisation de l'Oubangui. Aujourd'hui, comme au jour de mon ordination, je reste fermement décidé à faire tout ce qui dépend de moi pour contribuer au progrès moral, intellectuel et social du pays qui est le mien. En cela je ne crois pas m'éloigner du point de vue de l'Église catholique $»^{17}$.

Pour lui, il s'agissait d'effectuer les activités sacerdotales normales : messes, confessions, catéchismes; beaucoup de son temps était pris par les tournées en brousse, dans les postes délaissés par les missionnaires mobilisés. Parmi les postes secondaires sous sa juridiction, Boganda préférait Grimari, dont la situation était particulière : la Mission avait été dirigée avec plus ou moins de dévouement par ses prédécesseurs spiritains, avant d'être abandonnée au profit de l'Église protestante. Boganda retroussa ses manches, fit du porte-à-porte, séduisit non seulement les déserteurs mais aussi les fidèles protestants de longue date. La paroisse catholique connaissait une animation inédite. Le catéchisme reprenait partout. L'église était pleine les dimanches et les jours de fête. Des embryons d'entreprises économiques surgissaient : plantations de manioc, de bananes et autres. Des ateliers de fabrication de meubles en rotin produisaient régulièrement. Toutes ces choses étaient vendues et l'argent reversé à la procure de Saint-Paul à Bangui pour le compte de la Mission de Grimari. L'administrateur Dieu, chef de la subdivision de Grimari faisait part de ses inquiétudes à Mgr Grandin, sur le comportement de ce prêtre noir : il trouvait scandaleux que Boganda ait des idées autonomes alors que tout indigène devait mettre en pratique les idées de la colonisation. À ces remarques, Mgr Grandin répondait sèchement : «Il n'y a malheureusement pas de raison pour l'empêcher de penser selon une logique par ailleurs inattaquable !» (Siango 2004 : 145).

En 1944, au nom de la Mission de Grimari, Boganda présenta sa liste de besoins au père chargé de la procure à Bangui. Il se heurta à une fin de non-recevoir. Le père procureur 
lui fit remarquer que, prêtre séculier, il n'avait pas le droit de s'adresser à la procure des Spiritains pour faire face à ses besoins et à ceux de sa Mission. Boganda répondit simplement: "Vous avez raison! En effet, je ne suis pas Spiritain! Comment ai-je pu oublier ça? » (ibid.). L'abbé Boganda demanda alors à Mgr Grandin la restitution de l'argent qu'il avait versé à la procure pour le compte de la Mission de Grimari, afin de pouvoir se ravitailler pour un an avant la prochaine retraite. Interloqué, Monseigneur s'informa sur ce qui s'était passé. Il convoqua le responsable de la procure et lui intima l'ordre d'honorer, illico presto, la liste de Boganda.

31 La même année, alors que Boganda prenait ses congés à Bangassou, les Spiritains tinrent conseil et décidèrent de mettre fin aux humiliations que leur infligeait la méthode Boganda : il avait réussi à Grimari où les Spiritains avaient échoué. Il s'était aussi permis d'humilier le père procureur en exigeant la restitution de son argent, gagné grâce aux entreprises autonomes de Grimari. Ils envisagèrent alors de remplacer Boganda par deux prêtres spiritains. Pendant sa tournée à l'Est de son vicariat apostolique, Mgr Grandin s'arrêta à Grimari et fut déçu de ne pas y voir son protégé. Il le retrouva à Bangassou et lui reprocha d'avoir abandonné sa Mission. Boganda lui présenta le télégramme qui l'affectait à Bangassou. Mgr Grandin protesta avec véhémence déclarant que ce document n'était pas de lui. Il était effectivement l'œuvre du père Hemme, le vicaire général de Monseigneur et du père Morandeau, l'un des missionnaires influents de l'Oubangui. À tout cela, Boganda répondit que le télégramme portait le nom de l'évêque à qui il avait fait vœu d'obéissance. Il avait donc l'obligation de s'y conformer.

Cette affaire fut l'une des principales causes de la rupture de Boganda avec les Spiritains installés en Oubangui, et fut rappelée dans la réponse que Boganda envoya à Mgr Cucherousset le $1^{\mathrm{er}}$ décembre 1949 à la suite de la suspension qui lui avait été signifiée.

«La vraie cause, c'est l'esprit dominateur et esclavagiste de votre congrégation, c'est votre racisme, c'est le fait que vous vous êtes joints à nos oppresseurs pour nous maintenir dans l'ignorance et nous exploiter; ce sont les scandales nombreux que je vous ai dénoncés; la vraie cause enfin et toute personnelle celle-là, est le mensonge du Père Hemme me déplaçant de Grimari en me disant qu'il s'agissait d'une décision de l'Evêque ; C'est le Père Morandeau qui m'a volé à Grimari ; c'est Mgr Grandin qui m'écrivait : le noir n'est pas susceptible d'amélioration; ce sont les pères de Mbati qui ont raconté à Messieurs F. Serrand et R. Chevalier que les Noirs étaient menteurs, voleurs, paresseux, qu'il n'y avait rien à faire avec eux » (Penel 1995 : 241).

Même dans le milieu des Spiritains, le père Hemme n'avait pas bonne réputation. Son confrère, le père J. Bouchaud, écrivait sur les problèmes de Boganda, à son arrivée à Paris en décembre 1946, dans une lettre du 5 mai 1965 :

« Ayant été moi-même en Oubangui et ayant entendu parler de lui par des confrères qui l'avaient bien connu et vu à l'œuvre, je crois que ce qu'en écrit l'Abbé Boganda est malheureusement vraisemblable. Le Père Hemme avait un tempérament irascible et, comme il fréquentait beaucoup les coloniaux de Bangui, sa mentalité, son comportement et son langage s'en ressentaient. Des confrères m'ont dit, en fait, avoir été choqués de la façon dont le père traitait l'Abbé, même en public, alors que celui-ci était déjà prêtre et chargé de son ministère. Il est bien certain que certains missionnaires, et pas seulement en Oubangui, donnaient l'apparence d'être “colonialistes" ou négrophiles ${ }^{18}$.

Boganda, dans le $\mathrm{n}^{\circ} 3$ de son Bulletin mensuel d'action politique, économique et sociale en AEF, parle de négrophobes partout en Oubangui, 
"jusque dans les églises, à la communion et à l'autel, et certaines prétendues maisons d'éducation religieuse sont de véritables camps de concentration où les jeunes gens et jeunes filles sont soumis à des châtiments corporels exagérés, contrairement aux termes de la Constitution et respect de la personne humaine $»^{19}$.

Selon Jean-Dominique Penel (1995: 56), les causes de la rupture entre l'Église catholique de l'Oubangui et Barthélemy Boganda seraient liées à la foi de ce dernier et à la doctrine chrétienne. En effet, le 25 novembre 1949, Mgr Cucherousset suspendait officiellement Boganda qui ne pouvait plus, dès lors, exercer ses fonctions sacerdotales en public ni porter la soutane. Au demeurant, suspension ne signifie pas exclusion de l'Église. Boganda répondit en développant les griefs qu'il nourrissait à l'égard de l'Église catholique en Oubangui, particulièrement vis-à-vis des missionnaires :

«J'ai été suspendu par mesures politiques, racistes et arbitraires beaucoup plus que religieuses. Et vous avez ainsi fait le jeu du colonialisme et de la réaction dont nos missions ont toujours été, hélas, le plus ferme bastion. Vous avez été prompt à me frapper de peines canoniques que j'ai d'ailleurs méritées, je l'avoue encore une fois. Mais la responsabilité m'en incombe-t-elle entière? Si dans nos missions on ne m'avait pas exaspéré par des attitudes, des injustices, des injures dont "sale cochon de nègre" n'est qu'un exemple entre mille, je n'aurais peut-être jamais songé à vivre avec une française de la métropole pour contrarier mes confrères racistes et ils sont légion $»^{20}$.

Le racisme, Barthélemy Boganda le vivait durement et régulièrement. Il le dénonçait autant qu'il le pouvait. Le 28 octobre 1947, il adressa une lettre au père Morandeau, supérieur de la Mission Saint-Paul, à la suite d'une doléance d'un ouvrier de cette Mission ayant été maltraité par la police sous l'instigation du frère Mutien.

«Les oubanguiens ne demandent pas aux métropolitains la charité qui est une chose rare, ni l'amour puisque nous n'en sommes pas dignes. Mais la justice la plus élémentaire, celle que la France est venue apporter, où la trouverons-nous sinon dans nos missions? Aurait-on accompli ce geste à l'égard d'un européen ? ${ }^{21}$.

Parmi les questions de fond ayant poussé Barthélemy Boganda à la rupture, il y avait celle du célibat des prêtres qui lui paraissait avoir été imposé ni par les textes saints ni par les premiers successeurs de Jésus, mais par l'institution ultérieure à l'Église.

«Le Bon Dieu ne nous récompensera pas pour avoir fait des vœux ou fait semblant de les pratiquer. J'estime qu'il est plus digne de vivre avec une femme, que de faire un vœu auquel on manque constamment. Car le peuple aéfien n'est pas dupe ! Nul n'a jamais cru à notre chasteté et il y a certainement plus de scandale à accrocher une femme souvent à l'occasion du Ministère de la Confession que d'en avoir chez soi, officiellement, au vu et au su de tous $»^{22}$.

Il avait même menacé d'aller s'expliquer au Vatican. Barthélemy Boganda se maria le 13 juin 1950 devant l'officier d'état civil de Montmorency avec mademoiselle Jourdain, sa secrétaire, alors qu'un enfant était attendu. L'Église, après hésitations et peut-être l'attente d'un repentir, intervint pour rejeter l'abbé de son sein.

Par circulaire du 25 décembre 1949, Mgr Cucherousset informait les fidèles des sanctions frappant l'abbé Boganda : interdiction d'exercer les fonctions du sacerdoce, privation du port de l'habit ecclésiastique. Dans la réponse à la lettre de suspens du $1^{\text {er }}$ décembre 1949, l'abbé Boganda écrivait avoir suspendu sa soutane, partir à l'Assemblée nationale en civil et déclarait " pour moi, l'habit ne fait pas le moine, la soutane ne fait pas l'apôtre ni le prêtre. Je reste l'apôtre de l'Oubangui et de l'Église $»^{23}$.

Une fois les ponts ainsi coupés avec les missionnaires de l'Oubangui-Chari, Boganda n'eut pas le sentiment d'avoir rompu avec sa foi et ses idéaux religieux. Dans ses prises de 
position politiques fondamentales et dans ses options, il resta toujours profondément imprégné de la doctrine de l'Église. Il rompit avec des personnes, et en partie seulement avec une institution; il ne rompit pas avec les principes doctrinaux. Boganda donna délibérément à sa pratique politique une coloration religieuse et mystique.

À sa mort, cela lui a été reconnu. Devant le parvis de la cathédrale de Bangui où le père Ferraille donnait l'absoute (prière de la bénédiction finale), une étole était posée sur le cercueil, signe de son sacerdoce. Même Mgr Cucherousset, ce prélat qui mena la vie dure à Boganda, exprimait ses regrets le 2 mai 1962 dans une circulaire adressée à tous les missionnaires, suite à la loi supprimant à toutes les confessions religieuses leur droit à disposer d'établissements scolaires et à y dispenser l'enseignement. Le mot " unification » dans le texte de la loi, était pratiquement l'euphémisme de "nationalisation». Cette mesure privait l'Église catholique d'un champ d'apostolat qu'elle avait toujours privilégié et qui avait fait ses preuves dans la formation des anciens cadres du pays. Dans la circulaire, l'archevêque de Bangui déplorait « une mesure bien triste qui va à l'encontre des droits de l'Église ». Il y reconnaissait également la foi réelle et inébranlable de Boganda :

«Maintenant mieux que jamais, nous sentions combien grande a été la perte du Président Boganda. S'il a erré dans sa conduite personnelle, nous savons combien il aimait l'Église, combien il respectait ses droits. Il sentait dans l'Église la gardienne de la foi, la maîtresse de la doctrine et de la morale, la formation des consciences, le guide sûr et éclairé hors duquel il n'est pas facile de faire un bon chemin sur la terre. C'est pourquoi il rêvait d'une Afrique chrétienne. Dieu ait son âme et prions pour lui. »

Boganda, premier prêtre oubanguien, était fils de sorcier. Aux yeux du peuple, il participait des deux puissances. Il était le plus instruit des fils du pays ; prêtre, il était en rapport avec le monde invisible, il pouvait découvrir des choses cachées qui maintenaient les indigènes dans un état d'infériorité. Marié à une Blanche, il devenait l'égal des Blancs, c'était un homme hors du commun. La question des rapports entre Boganda et l'Église doit tenir compte de tous ces éléments qui s'entremêlaient étroitement et dont Boganda était très conscient, comme nous l'avons souligné tout au long de l'exposé.

La résurgence du souvenir et l'amplification des relations conflictuelles avec ses supérieurs et ses collègues religieux d'Oubangui, sa pratique sacerdotale, sa relation avec mademoiselle Jourdain, sa secrétaire qui deviendra son épouse légitime, ont été autant d'éléments ayant caractérisé les rapports entre Boganda et l'Église catholique en Oubangui.

\section{BIBLIOGRAPHIE}

Augouard, P. P.

193444 années au Congo : suite de « 28 années » et « 36 années » au Congo et fin des lettres de Mgr Augouard, 1905-1914-1921, Évreux, Poussin. 
DE BANVILle, G.

2000 Kalouka et Zoungoula, les deux premières religieuses de Brazzaville, au Congo, 1892-1909, Paris, Karthala (« Mémoire d'Églises »).

CALLOC'H, J.

1911a Vocabulaire français-sango et sango-français : langue commerciale de l'Oubangui-Chari, Congo français, Paris, P. Geuthner.

1911 b Vocabulaire gmbwaga-gbanziri-mondjombo, avec grammaire, Paris, P. Geuthner.

1911c Vocabulaire français-gbéa, Congo français, Paris, P. Geuthner.

COQUERY-VIDROVITCH, C.

1972 Le Congo au temps des grandes compagnies concessionnaires, 1898-1930, Paris-La Haye, Mouton («Le Monde d'outre-mer passé et présent, $1^{\text {re }}$ série, Études, 37 »).

DE WiTTE, J.

1924 Un explorateur et un apôtre au Congo français, Mgr Augouard : sa vie, ses notes de voyage et sa correspondance, Paris, Émile-Paul.

ERNOULT, J.

1995 Les Spiritains au Congo de 1865 à nos jours, Paris, Congrégation du Saint-Esprit (« Mémoire spiritaine, études et documents »).

KALCK, P.

1995 Barthélemy Boganda, 1910-1959, élu de Dieu et des Centrafricains, Saint-Maur-des-Fossés, Éditions Sépia (« Sépia poche »).

KinATA, C.

2004 « Les Administrateurs et les Missionnaires face aux coutumes au Congo français », Cahiers d'Études africaines, XLIV(3), 175 : 593-607.

MERLE, M. (dir.)

1967 Les églises chrétiennes et la décolonisation, Paris, Armand Colin (« Cahiers de la Fondation nationale des sciences politiques, $151 »)$.

MORIZUR, J.

1948 La vie d'un petit séminaire en Afrique Noire, Paris, Union missionnaire du clergé en France.

NIDA, E. A.

1954 Customs and Cultures. Anthropology for Christian Missions, New York, Harper \& Row Publishers.

NZABAKOMADA-YAKOMA, R.

1986 L'Afrique Centrale insurgée. La guerre du Kongo-Wara 1928-1931, Paris, L'Harmattan.

PENEL, J.-D.

1995 Barthélemy Boganda, écrits et discours. 1946-1951 : la lutte décisive, Paris, L'Harmattan.

SIANGO, B. B.

2004 Barthélemy Boganda, premier prêtre oubanguien fondateur de la République centrafricaine, Pierrefitte-sur-seine, Bajag-Meri.

\section{NOTES}

1. Lettre de Mgr Grandin au père Lecomte, Bangui le 19 Octobre 1946, citée dans PENEL (1995: 105). 
2. Lettre de Barthélemy Boganda au père Féraille en congé en France, Paris, le 30 avril 1947, citée dans PenEl (ibid. : 134).

3. Cité dans PenEl (1995: 21).

4. Mgr Carrie avait ordonné les deux premiers prêtres indigènes de son vicariat le 17 décembre 1892.

5. Mgr Augouard, Rapport quinquennal de 1920 à la Sacrée Congrégation de la Propagande à Rome, cité dans DE WiTTE (1924 : 346).

6. Rapport annuel du séminaire Saint-Paul de Kibouende, Arch Cssp B522.A, Vicariat apostolique de Brazzaville, 1937.

7. Ibid.

8. Ibid.

9. Projet d'établissement du petit séminaire à Kibouende par le Père Ramaux, Arch Cssp B J3.26A, 1939.

10. Ibid.

11. Bulletin général, avril 1913, cité dans ERnOULT (1995 : 225).

12. Instruction «Quo Efficacius » de Benoît XV du 6 janvier 1920, citée dans MERLE (1967 :

76).

13. Mgr CALLOC'H (1911a, 1911b, 1911c) a publié plusieurs vocabulaires. Il a traduit plusieurs catéchismes dans les langues oubanguiennes, dont un en ngbaka. Les archives des Spiritains, à Chevilly, conservent son très volumineux Vocabulaire français-ngbaka en deux tomes.

14. É. Cointet, Lettres à sa famille, citée dans COQUERY-VIDROVITCH (1972 : 209).

15. Rapport de l'inspecteur du contrôle local, 30 avril 1907, cité dans COQUERY-VIDROVITCH (1972: 129).

16. Lettre de Boganda à son évêque, Bambari, le 21 décembre 1943, citée dans SIANGO (2004: 122).

17. Lettre de Boganda à son évêque, citée dans Pierre KALCK (1995 : 58).

18. Lettre du père J. Bouchaud, chargé de Revue Annales Spiritaines, en témoignage sur les problèmes de Boganda à son arrivée à Paris en décembre 1946, citée dans PENEL (1995 : 389).

19. Barthélemy Boganda, « Pour sauver un peuple », Bulletin mensuel d'action politique, économique en AEF, $\mathrm{n}^{\circ}$ 3, 1948, cité dans PENEL (1995 : 196).

20. Lettre de Barthélemy Boganda à Mgr Cucherousset, évêque de Bangui, Paris le $1^{\mathrm{er}}$ décembre 1949, citée dans Penel (ibid. : 237).

21. Lettre au père Morandeau, supérieur de la Mission Saint-Paul, le 28 octobre 1947, citée dans PENEL (ibid. : 155).

22. Lettre à Mgr Cucherousset, évêque de Bangui, Paris, $1^{\mathrm{er}}$ décembre 1949, op. cit.

23. Ibid. 


\section{RÉSUMÉS}

Barthélemy Boganda, originaire de la Lobaye en pleine forêt équatoriale, devenait le premier prêtre catholique oubanguien le 27 mars 1938. Orphelin très tôt, ses parents ayant été tués par les miliciens, il fut recueilli par les missionnaires catholiques de la congrégation du Saint-Esprit. Ses tuteurs le poussèrent à faire de la politique en le faisant élire député de l'Oubangui-Chari à l'Assemblée de l'Union française en 1946. C'était l'objectif de Boganda depuis son baptême: défendre les intérêts des Oubanguiens qui étaient considérés par les Blancs comme moins que rien. Boganda, le plus instruit des fils du pays avait une très forte réputation fondée sur trois éléments : prêtre, il était censé être en rapport avec le monde invisible; fils de sorcier, il était craint. Il était ainsi censé être capable de découvrir des choses cachées maintenant les indigènes dans un état d'infériorité d'où ils espéraient sortir. Enfin, marié à une Blanche, il devenait l'égal des Blancs. La question des relations entre Boganda et l'Église doit tenir compte de tous ces éléments qui s'entremêlaient étroitement. Boganda lui-même en était très conscient. La résurgence du souvenir des compagnies concessionnaires martyrisant les populations grâce à l'appui de l'administration coloniale, l'amplification des relations conflictuelles dues au racisme de ses supérieurs et de ses confrères religieux spiritains, sa pratique sacerdotale avaient par ailleurs largement orienté les rapports entre Boganda et l'Église catholique.

Barthélemy Boganda and the Catholic Church in Oubangui-Chari. - Barthélemy Boganda, born in the Lobaye basin in the Equatorial forest, became the first ever Oubanguian Catholic priest on 27 March 1938. After his parents were killed by militia when he was still very young, he was adopted by Catholic missionaries of the Holy Spirit congregation. They encouraged him to become a politician and run for election to the National Assembly of France, where he was elected in 1946. Thus, Boganda was able to fulfil his life-long dream of fighting for equals rights for Oubanguians. The most educated son of the country had a very strong reputation based on three elements: as a priest, he was supposed be in contact with the invisible world; as a sorcerer, he was feared-he was deemed capable of discovering what kept indigenous people in the state of inferiority from which they wanted to extract themselves; finally, as the husband of a white woman, he had become an equal of the whites. An analysis of the relation between Boganda and the Church must take into account all these intertwined elements, and Boganda himself was very aware of this. The resurging memory of concession companies martyring people with the support of the colonial administration, the increasingly conflictual relations resulting from the racism of his superiors and fellow Spiritans, and his sacerdotal practice are all elements that have substantially influenced his relationship with the Catholic Church.

\section{INDEX}

Mots-clés : Église catholique, racisme, Oubangui-Chari, Boganda Barthélemy, administration coloniale, Assemblée de l'Union française, congrégation du Saint-Esprit, prêtre

Keywords : Catholic Church, racism, colonial government, congregation of Saint-Esprit, priest 


\section{AUTEUR}

\section{CÔME KINATA}

École normale supérieure, Brazzaville. 